2018-03-12

\title{
INVESTIGATION OF TEMPERATURE RESPONSE OF PHOTOPOLYMER MATERIAL USED FOR HOLOGRAPHIC SENSOR
}

\author{
Muhammad Irfan \\ Technological University Dublin, d15128418@mytudublin.ie \\ Tatsiana Mikulchyk \\ Technological University Dublin, Tatsiana.mikulchyk@tudublin.ie \\ Suzanne Martin \\ Technological University Dublin, suzanne.martin@tudublin.ie
}

See next page for additional authors

Follow this and additional works at: https://arrow.tudublin.ie/scschphycon

Part of the Optics Commons, and the Polymer Chemistry Commons

\section{Recommended Citation}

Naydenova, I. et al (2018) "Investigation of temperature response of photopolymer material used for holographic sensor," 15th International Bhurban Conference on Applied Sciences and Technology (IBCAST), Islamabad, 2018, pp. 22-27, doi: 10.1109/IBCAST.2018.8312179.

This Conference Paper is brought to you for free and open access by the School of Physics \& Clinical \& Optometric Science at ARROW@TU Dublin. It has been accepted for inclusion in Conference Papers by an authorized administrator of ARROW@TU Dublin. For more information, please contact arrow.admin@tudublin.ie, aisling.coyne@tudublin.ie,gerard.connolly@tudublin.ie. Funder: Dublin Institute of Technology Fiosraigh Postgraduate Scholarship Fund

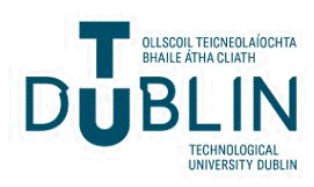




\section{Authors}

Muhammad Irfan, Tatsiana Mikulchyk, Suzanne Martin, and Izabela Naydenova 


\title{
INVESTIGATION OF TEMPERATURE RESPONSE OF PHOTOPOLYMER MATERIAL USED FOR HOLOGRAPHIC SENSOR
}

\author{
M. Irfan, T. Mikulchyk, S. Martin, I. Naydenova* \\ Centre for Industrial and Engineering Optics/School of Physics and Clinical \& Optometric Sciences, \\ College of Sciences and Health, \\ Dublin Institute of Technology, Kevin Street, Dublin 8, Ireland. \\ E.mail: izabela.naydenova@dit.ie
}

\begin{abstract}
Recently, functionalised photopolymer has emerged as a versatile recording material in the field of optical holography due to its novel characteristics and potential use in the development of holographic based sensors, and optical elements. This work describes the temperature response of a newly developed photopolymer, containing of a monomer $\mathrm{N}$ isopropylacrylamide (NIPA) which under photopolymerisation forms a temperature sensitive polymer - Poly(Nisopropylacrylamide) (PNIPA). The photonic sensor was developed by holographic recording of volume phase transmission gratings in a self-processing NIPA-based polymer with a $532 \mathrm{~nm}$ laser beam. A $633 \mathrm{~nm}$ probe beam was used to monitor real time diffraction efficiency (DE) growth curve and the temperature dependent response of $\mathrm{DE}$ in the temperature range $22-50{ }^{\circ} \mathrm{C}$. It was observed that the $\mathrm{DE}$ increased with increasing temperature at $10{ }^{\circ} \mathrm{C} \mathrm{min}^{-1}$ and started to drop by reversing the temperature at $5{ }^{\circ} \mathrm{C} \mathrm{min}^{-1}$. It was also observed that the response depends on the rate of heating/cooling and the time spent at elevated temperature. The observed response to temperature could be used to design an indicator for packaging, showing that the content of the package has been exposed to a temperature that was above a predetermined temperature limit.
\end{abstract}

Key Words: Holographic sensor, Photopolymer, Volume phase transmission gratings

\section{INTRODUCTION}

In optical holography photopolymers are some of the most commonly used recording media. In recent years the research interest in photopolymers for holography has grown due to their strong potential to be used in the development of holographic sensors. Compared to other recording materials, photopolymers are considered as attractive materials for fabrication of holographic sensors due to their ability to be functionalised, large dynamic range, higher sensitivity, selfprocessing nature and relatively low production cost. Photopolymers have been intensively studied materials due to possible practical applications in holographic data storage, holographic optical elements, and holographic sensors. [1-7].

Among the various applications, holographic sensor is a relatively recent application of photopolymer materials. Holographic sensors are functionalised photonic structures which are developed by holographic recording [7-9]. In its simplest form the holographic sensor is a holographic diffraction grating embedded in the photopolymer material, which has all the necessary features to work as a sensor. The sensor can change its properties, such as diffraction efficiency (DE) or spectral response, upon the interaction with a target analyte. Their ability to display visual information in the presence of some stimulus (physical or chemical) is what makes holographic sensors suitable for applications ranging from medical diagnostics to environmental sensing and security. Holographic sensors have some advantages over the other sensors including feasibility for large scale production, lightweight, and possibility to be multiplexed and miniaturised [7].

N-isopropylacrylamide (NIPA)-based photopolymer has recently been developed and optimised for holographic recording both in the transmission and reflection modes [10]. The main approach used in the development of this photopolymer is the introduction of low toxicity and temperature sensitive monomer NIPA instead of acrylamide, which is often used [5-6, 11-12]. This newly developed polymer has a good recording capability and greater sensitivity to temperature. The temperature sensitivity of PNIPA is due to its phase transition that occurs at a critical temperature. PNIPA has a lower critical solution temperature (LCST) at $\sim 32{ }^{\circ} \mathrm{C}$. The LCST can be varied by the copolymerisation with other appropriate monomers. It is expected that the copolymerisation may lead to a change in the optical response and to enhanced sensitivity of the devices.

In this work, the temperature dependent response of a newly developed photopolymer, containing a temperature sensitive monomer NIPA, has been described. This work will help to understand the temperature response of a NIPA-based optical sensor for practical applications, such as material packaging and security.

\section{THEORETICAL BACKGROUND}

Based on the recording geometry there are two types of holographic gratings - transmission and reflection, both can work as a sensor. Transmission grating is recorded when both the recording beams approach the photosensitive medium from the same side, while reflection grating is recorded when both the beams approach the photosensitive medium from the two opposite sides. Here, un-slanted transmission gratings are produced by placing the photosensitive medium in the region of overlapping of two coherent recording beams, such that the wave vectors of the beams are making opposite and equal angles with the surface normal of the medium. The spatial frequency of the transmission holographic grating is determined by the angle between the two recording beams and is given by: 


$$
2 \Lambda \sin \theta=\lambda
$$

Where $\Lambda$ is the fringe spacing and is $\Lambda=1 / \mathrm{F}$ (here $\mathrm{F}$ is spatial frequency), $\theta$ is the Bragg angle and $\lambda$ is the wavelength of recording beams.

\section{A. Mechanism of holographic grating formation in photopolymer}

When the photopolymer layer is exposed to an interference pattern generated by recording beams having suitable wavelength, a polymerisation process is started in the illuminated areas where monomer molecules form polymer chains leading to change in molecular polarisability and density. The photopolymerisation is accompanied by diffusion processes. Due to the generated concentration gradient, monomers will diffuse from dark to bright fringe regions and short/mobile polymer chains will diffuse in opposite direction. Holographic recording in photopolymers leads to a spatial variation of the refractive index of the material due to photopolymerisation and diffusion of photopolymer components. A volume phase grating is recorded by means of a spatial variation of the refractive index and/or thickness, as shown in Fig.1.

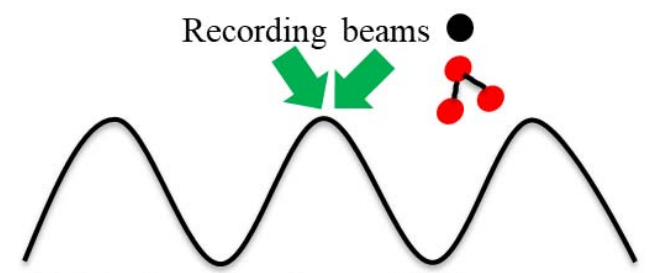

(a) Interference pattern of light

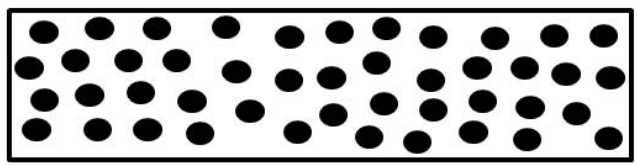

(b) Photopolymer before light exposure

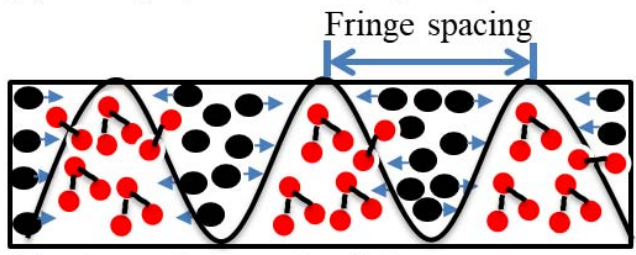

(c) Photopolymer after light exposure

Figure 1. Schematic diagram of holographic grating recording process in photopolymer.

\section{B. Principle of operation of a holographic sensors}

The working principle of a holographic sensor is shown in Fig. 2. All holographic sensors utilise the same optical phenomena which is independent of analyte which the sensor detects. In order to use the response of hologram for the development of the sensor, it is very important to understand how the response is produced. When the analyte sensitive polymer layer (in which the grating is recorded) is exposed to the analyte, changes in the fringe spacing and the refractive index modulation of the photonic structure occur. The sensor signal is received as change in DE, Bragg angle and peak wavelength (if probed with broad spectral range source).

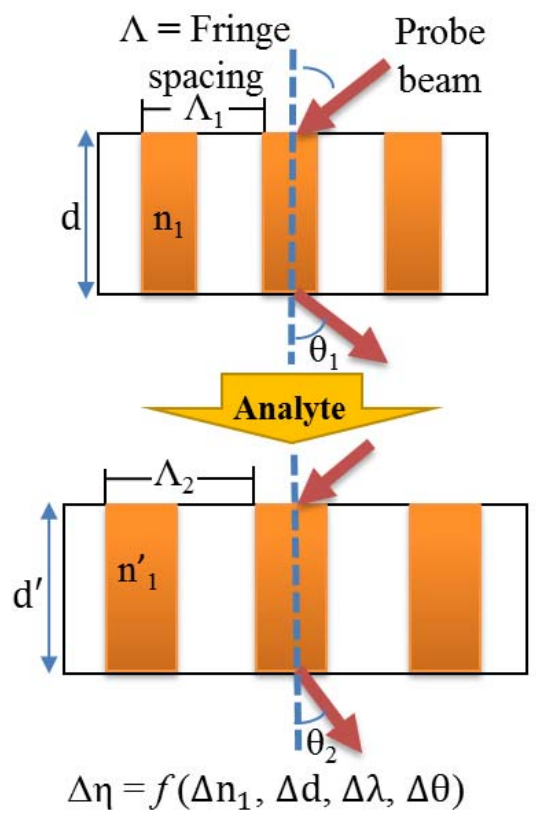

Figure 2. A diagrammatic view of the operation principle of sensor based on holographic transmission grating.

\section{Characterisation of a holographic sensor}

The gratings that are recorded can be either entitled as thick (volume) or thin (plain) gratings. One can easily use the value of Q-factor in order to categorise them into thick or thin. For the holographic gratings which have $\mathrm{Q}>10$ are considered as thick gratings while those having $\mathrm{Q}<1$ are considered as thin gratings. When $1<\mathrm{Q}<10$ the regime is intermediate and you may have one or the other depending on the refractive index modulation. For the volume phase grating regime the value of $\mathrm{Q}$-factor corresponding to $\mathrm{Q}>10$ is given as [13]:

$$
\mathrm{Q}=2 \pi \lambda \mathrm{d} / \mathrm{n}_{0} \Lambda^{2}
$$

Where $\lambda$ is the recording beam wavelength, $d$ is the thickness of the polymer layer, $\mathrm{n}_{0}$ is the average refractive index of the photosensitive medium and $\Lambda$ is the fringe spacing (grating period).

In the present research work the value of Q-factor was calculated for the recorded transmission gratings with a spatial frequency of 830 lines $/ \mathrm{mm}$ and the thickness of the polymer layer used $80 \pm 5 \mu \mathrm{m}$. Hence, the value of Q-factor obtained is in the range of 115 to 130 , which clearly justifies the application of coupled wave theory [14] for the calculation of DE which is given by:

$$
\eta=\sin ^{2}\left(\pi \mathrm{n}_{1} \mathrm{~d} / \lambda \cos \theta\right)
$$

Here $\eta$ is the DE, $n_{1}$ is refractive index modulation, $d$ is the thickness of the grating, $\lambda$ is the probe (reconstructing) beam wavelength, $\theta$ is the Bragg angle inside the polymer layer.

When the transmission grating is exposed to an analyte, this will change the DE $(\Delta \eta)$ caused by the effect of different parameters which is obtained by differentiation of equation (3) and is given by [7]: 


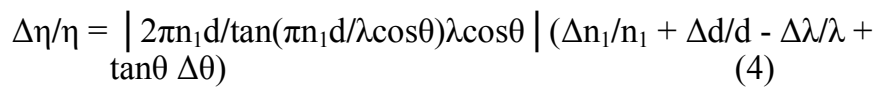

Where $\Delta \mathrm{n}_{1}$ represents the refractive index modulation change, $\Delta \mathrm{d}$ is the change in thickness of the layer, $\Delta \lambda$ is the variation in wavelength of probe beam and $\Delta \theta$ is the shift in Bragg angle. In the present work, the probe wavelength and the probe beam angle are kept constant, thus only the first two parameters - refractive index modulation and thickness influence the DE change.

\section{METHODS AND MATERIALS}

\section{A. Photopolymer solution preparation}

Photopolymer composition previously [10] optimised for holographic recording was used in this study. The chemical components are given in Table I. The chemicals were purchased from Fisher Scientific and Sigma Aldrich and used without any further purification.

TABLE I. THE AMOUNT OF CHEMICAL COMPONENTS USED IN PREPARATION OF PHOTOPOLYMER SOLUTION

\begin{tabular}{|c|c|}
\hline Components & $\begin{array}{c}\text { Molar } \\
\text { Concentration } \\
\text { in polymer solution }\end{array}$ \\
\hline (N-Isopropylacrylamide), NIPA & $0.097 \mathrm{M}$ \\
\hline (N,N'Methylenebisacrylamide), BA & $0.053 \mathrm{M}$ \\
\hline (Polyvinyl alcohol $10 \% \mathrm{w} / \mathrm{v}), \mathrm{PVA}$ & $8.79 \% \mathrm{w} / \mathrm{v}$ \\
\hline (N-Phenylglycine), NPG & $0.0145 \mathrm{M}$ \\
\hline (Erythrosine B dye 0.11 \%w/v), Er B & $1.37 \times 10^{-4} \mathrm{M}$ \\
\hline Glycerol & $0.15 \mathrm{M}$ \\
\hline
\end{tabular}

To obtain a $10 \% \mathrm{w} / \mathrm{v}$ PVA stock solution, $10 \mathrm{~g}$ of polyvinyl alcohol (molecular weight 9,000-10,000, $80 \%$ hydrolysed) was dissolved in $100 \mathrm{ml}$ deionised water using a magnetic stirring and heated in open ambient laboratory conditions at $75{ }^{\circ} \mathrm{C}$. Similarly, $0.11 \mathrm{~g}$ of Erythrosine B was dissolved in $100 \mathrm{ml}$ deionised water at room temperature by magnetic stirring to obtain a dye solution of the desired concentration $(0.11 \% \mathrm{w} / \mathrm{v})$.

For the preparation of photopolymer solution the optimised amount of all the components as given in [10], were mixed thoroughly with magnetic stirrer for 2 hours at ambient laboratory conditions (temperature $24 \pm 0.5{ }^{\circ} \mathrm{C}$ and relative humidity (RH) $38 \%$ ).

\section{B. Photopolymer layer preparation}

Photopolymer layers for holographic recording were prepared using $1.5 \mathrm{ml}$ of the solution which was evenly spread over on the microscopic glass slide of dimensions $7.6 \mathrm{~cm} \times 2.6$ $\mathrm{cm}$. The slides with the polymer layer were placed on a levelled surface and allowed to dry in the dark room for 18 hours at the $38 \% \mathrm{RH}$ and $24 \pm 0.5^{\circ} \mathrm{C}$.

\section{Holographic recording}

As shown in Fig. 3, a photonic structure was created by holographic recording of an un-slanted volume phase transmission gratings with spatial frequency of 830 lines $/ \mathrm{mm}$ in the NIPA based photopolymer. The two recording beams were obtained by splitting the laser light from a $532 \mathrm{~nm}$ $\mathrm{Nd}: \mathrm{YVO}_{4}$ laser by a polarising beam splitter. The polarisation of the transmitted beam was rotated with the help of a halfwave-plate in order to ensure that the two recording beams were s-polarised, thus providing conditions for maximum visibility of the recorded interference fringes. The sample was mounted on a computer controlled rotational stage (Newport ESP300) and recorded for $100 \mathrm{~s}$ with total recording intensity $3.8 \mathrm{~mW} / \mathrm{cm}^{2}$. The optimum intensity was previously determined by studying the intensity dependence of the DE [10]. A $633 \mathrm{~nm}$ He-Ne laser was used as a probe beam to monitor a real time growth curve of the DE given in Fig. 4(a). The Bragg selectivity curve measurement was performed by monitoring the diffracted beam intensity with an optical power meter (Newport Model 840) while the sample was rotated. After recording, the gratings were bleached with ultraviolet (UV) light for $100 \mathrm{~s}$ using a UV exposure intensity 60 $\mathrm{mW} / \mathrm{cm}^{2}$ provided by Dymax UV-curing system (model ECE200) to polymerise remaining monomers. In this experiment, DE was calculated as the ratio of the diffracted beam intensity $\left(\mathrm{I}_{\mathrm{d}}\right)$ and the incident beam intensity $\left(\mathrm{I}_{\mathrm{i}}\right)$. Fig. 4(b) shows Bragg selectivity curves of the diffracted beam acquired through LabVIEW software before and after the UV-curing. Only a small decrease of the DE was observed after UV postrecording curing. This indicates that the recorded grating is mainly due to density changes caused by concentration gradient driven monomer diffusion which occurs during the holographic recording process.

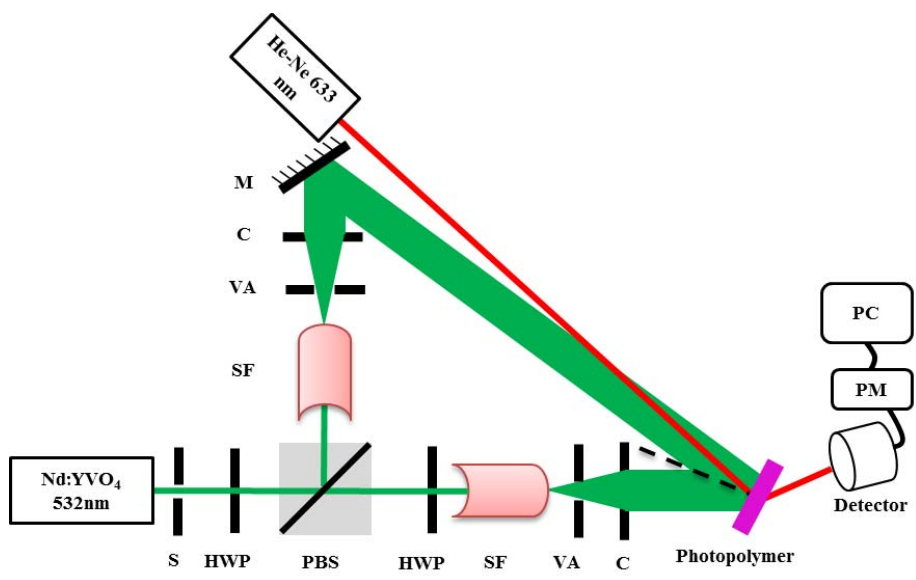

Figure 3. Experimental setup used for the recording of transmission holographic gratings. S-electronic shutter, HWP-half wave plate, PBSpolarising beam splitter, SF-spatial filter, VA-variable aperture, C-collimator, M-mirror, PM-power meter, PC-computer. 

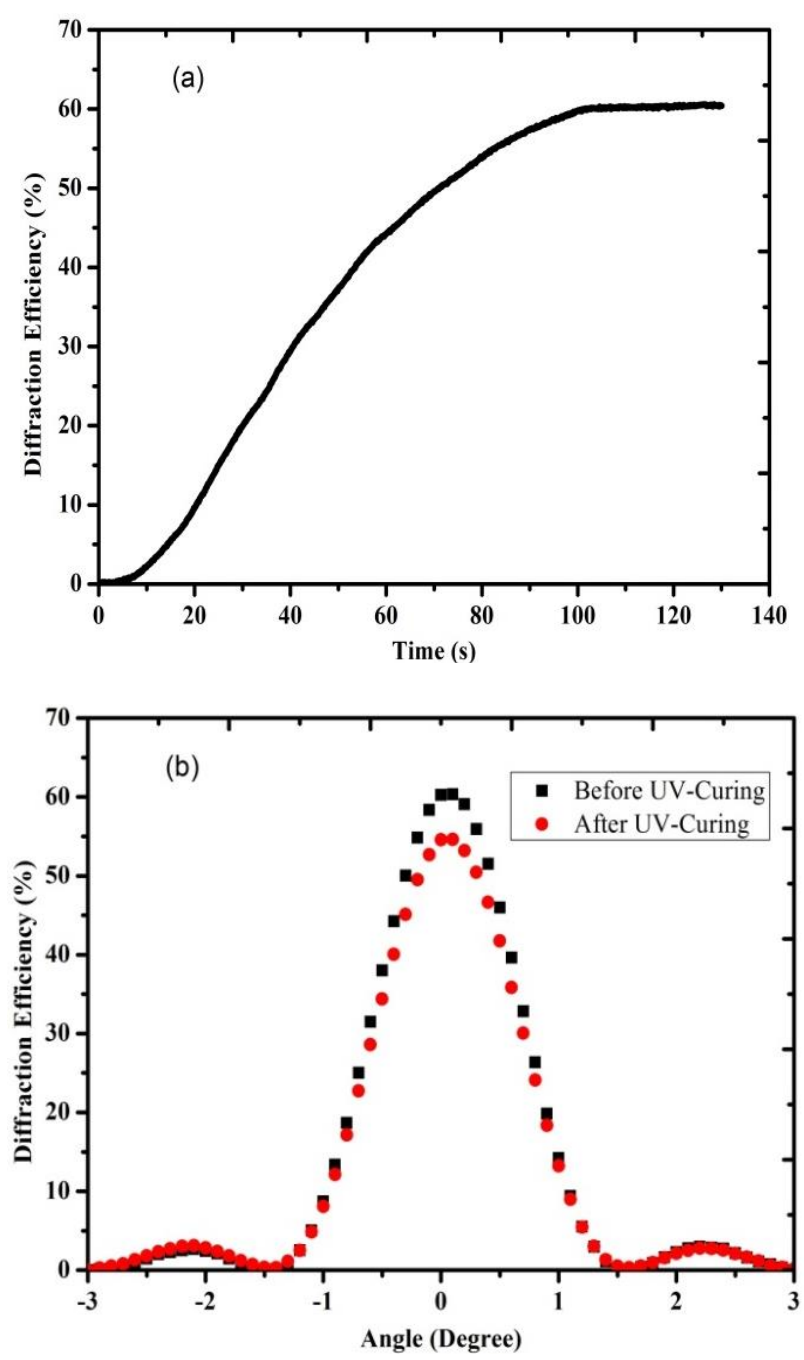

Figure 4. Characterisation of the holographic diffraction grating. (a) Real time growth curve of DE; (b) Bragg selectivity curve of DE for the photopolymer

\section{Temperature response}

The temperature response study of the developed photonic structure was carried out by exposing the structure to temperature in the range of $22{ }^{\circ} \mathrm{C}$ to $50{ }^{\circ} \mathrm{C}$, and by monitoring the DE. The experimental setup used for measurement is shown in Fig. 5. The sample was placed on the temperature controlled stage (Linkam Model THMS600) and the lid of the cell was tightly closed. The temperature was controlled in the required range using a temperature controller (Linkam Model TMS 93). Initially, temperature of the stage was increased from $22{ }^{\circ} \mathrm{C}$ to $50{ }^{\circ} \mathrm{C}$. Then cool air was pumped into the stage with a cooling pump (Linkam Model LNP) to decrease the temperature back to $22{ }^{\circ} \mathrm{C}$ to study reversibility of the structure. A dewar flask filled with ice was used to produce the cool air which was needed to decrease the stage temperature to $22{ }^{\circ} \mathrm{C}$. During the temperature exposure, the changes in the intensity of the first order diffracted beam $\left(\mathrm{I}_{1}\right)$ and intensity of the transmitted beam $\left(\mathrm{I}_{0}\right)$ were monitored simultaneously by two optical power meters (Newport Model $840)$. The DE in this experiment was defined by $\mathrm{I}_{1} /\left(\mathrm{I}_{1}+\mathrm{I}_{0}\right)$.

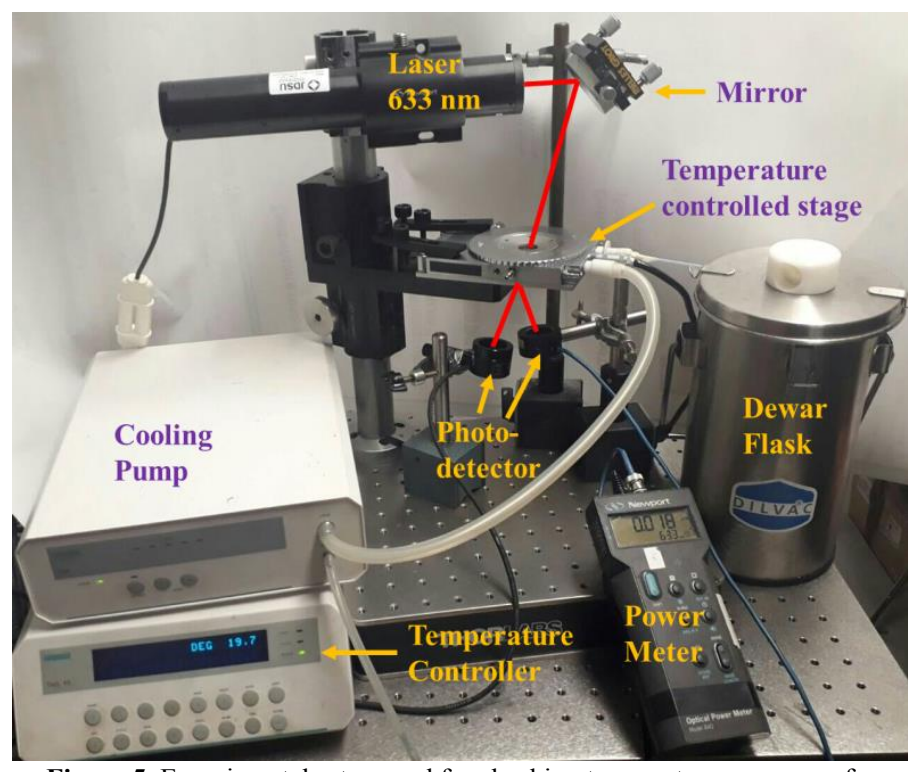

Figure 5. Experimental setup used for checking temperature response of recorded gratings.

\section{RESULTS AND DISCUSSION}

\section{A. Investigation of the dependence of temperature response on the rate of temperature change}

\section{Slow rate of temperature change}

Fig. 6 shows the temperature dependent response of the developed NIPA based photonics structure in the temperature range of $22{ }^{\circ} \mathrm{C}$ to $50{ }^{\circ} \mathrm{C}$, with increasing temperature at rate of $5{ }^{\circ} \mathrm{C} \mathrm{min}-1$ in the forward direction and at a decreasing rate of $1{ }^{\circ} \mathrm{C} \mathrm{min}{ }^{-1}$ in the reversed direction. Clearly, the DE increases at temperatures below $35{ }^{\circ} \mathrm{C}$ and decreases above $35{ }^{\circ} \mathrm{C}$. This behavior of the DE with the increasing temperature can be explained by the switching from hydrophilic to hydrophobic character of the photopolymer layer when the temperature changes from below LCST to above LCST. The LCST of pure PNIPA is at $32{ }^{\circ} \mathrm{C}$, and the change was observed close to this temperature. Below $32{ }^{\circ} \mathrm{C}$ photopolymer layer has a hydrophilic character and as the temperature increases the layer absorbs water molecules from air and this causes a swelling of the layer. Increase of the refractive index modulation due to different absorption of the water in the bright and dark fringe areas is also possible. At temperature above $35{ }^{\circ} \mathrm{C}$ a decrease in the DE is observed and this must be related to the hydrophobic character of PNIPA above LCST. Water molecules are expelled from the PNIPA rich areas and this causes shrinkage and decreases the refractive index modulation which leads to a decrease in the DE [10]. In this experiment the temperature of the stage was decreased immediately after reaching $50{ }^{\circ} \mathrm{C}$.

With decrease in temperature in the reversed direction the $\mathrm{DE}$ is still decreasing until reaching $35^{\circ} \mathrm{C}$. After that the DE remains almost unchanged. The normalised DE was defined as the ratio of the $\mathrm{DE}$ at the current temperature and the $\mathrm{DE}$ at the start of the experiment at $22{ }^{\circ} \mathrm{C}$. 


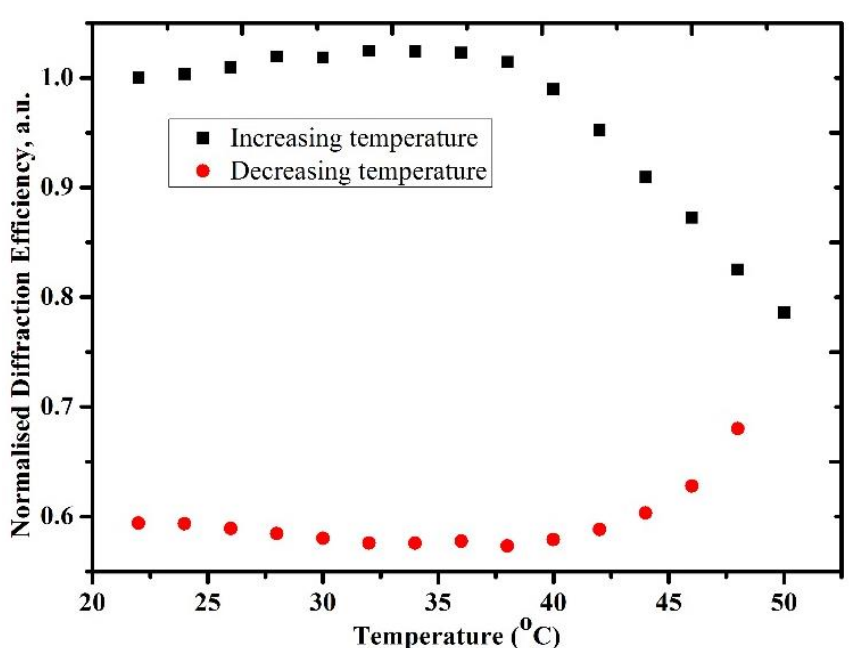

Figure 6. Normalised DE Vs Temp, temperature increased at rate $5{ }^{\circ} \mathrm{C} \mathrm{min}{ }^{-1}$ and decreased at rate of $1{ }^{\circ} \mathrm{C} \mathrm{min}$.

\section{Fast rate of temperature change}

The temperature response was also monitored at a faster rate of $10{ }^{\circ} \mathrm{C} \mathrm{min}^{-1}$ in the forward half cycle followed by the next half cycle in the reversed direction with a decreasing temperature rate of $5{ }^{\circ} \mathrm{C} \mathrm{min}^{-1}$. The $\mathrm{DE}$ response is displayed in Fig. 7. In this case it is expected that the polymer is only in the hydrophilic state as it does not achieve the LCST of PNIPA quickly enough until it reaches to a temperature above $45{ }^{\circ} \mathrm{C}$. At these temperatures $\mathrm{DE}$ continuously increases probably due to water molecules absorbed by the polymer. Above $45{ }^{\circ} \mathrm{C}$, the polymer achieves LCST and DE reaches saturation. The temperature of the stage was decreased immediately after reaching $50{ }^{\circ} \mathrm{C}$. During the reversed half cycle from $50-22{ }^{\circ} \mathrm{C}, \mathrm{DE}$ is still decreasing. $\mathrm{DE}$ is decreasing possibly due to the fact that the actual polymer temperature is still above LCST of the PNIPA, due to which it continues to expel water molecules. In order to check this hypothesis the layer was left at $50{ }^{\circ} \mathrm{C}$ for longer amount of time. Fig. 8 shows the measured DE when the layer was left for 10 min at $50{ }^{\circ} \mathrm{C}$.

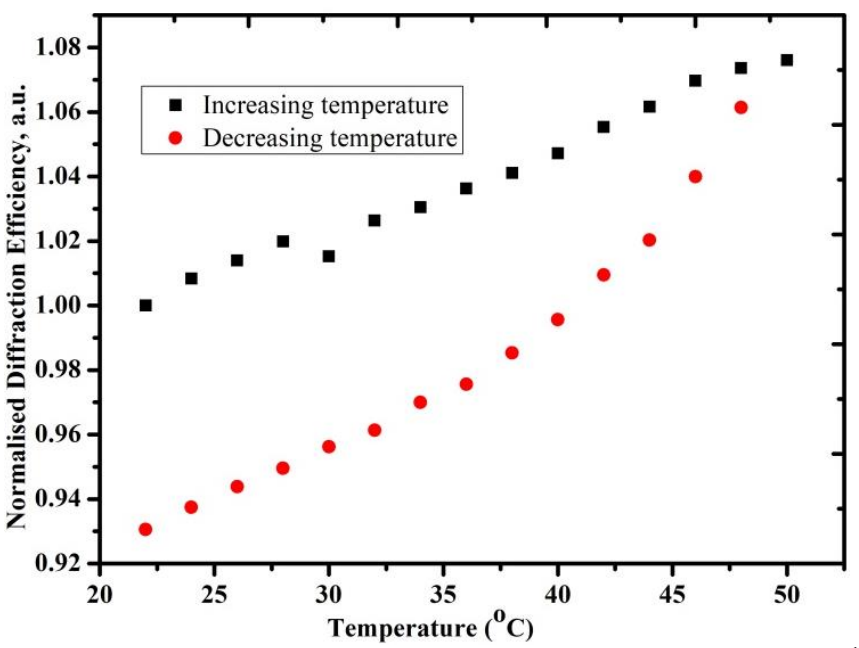

Figure 7. Normalised DE Vs Temp, temperature increased at rate $10{ }^{\circ} \mathrm{C} \mathrm{min}{ }^{-1}$ and decreased at rate of $5{ }^{\circ} \mathrm{C} \mathrm{min}^{-1}$. Temperature of the stage was decreased immediately after reaching $50{ }^{\circ} \mathrm{C}$.

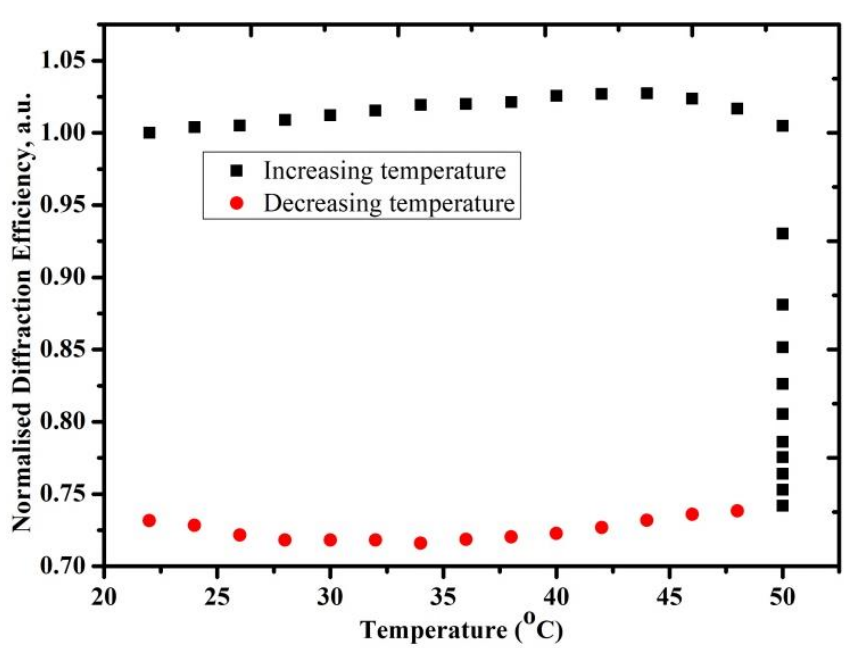

Fig 8. Normalised DE Vs Temp, temperature increased at rate $10{ }^{\circ} \mathrm{C} \mathrm{min}^{-1}$ and decreased at rate of $5{ }^{\circ} \mathrm{C} \mathrm{min}^{-1}$. Temperature of the stage was kept at $50{ }^{\circ} \mathrm{C}$ for $10 \mathrm{~min}$.

\section{B. Effect of exposure time at elevated temperature}

The effect of elevated temperature on the DE of gratings for different exposure time was studied by exposing the photonic structure to $50{ }^{\circ} \mathrm{C}$. The dependence of normalised DE on exposure time at $50{ }^{\circ} \mathrm{C}$ is shown in Fig. 9. The temperature controller was used to keep the samples temperature constant while varying the exposure time in the range of $1-10 \mathrm{~min}$. Clearly, for a constant exposure temperature $50{ }^{\circ} \mathrm{C}$, the rate of decrease of $\mathrm{DE}$ is different and seems approaching to saturation for a longer exposure time of $10 \mathrm{~min}$. As shown in Fig. 10, it was also observed that the irreversibility of the temperature induced changes depend on duration of the time spent at temperature above the LCST of the PNIPA. The irreversible changes in the DE were observed by exposing the structure to elevated temperature for the duration 1-10 min.

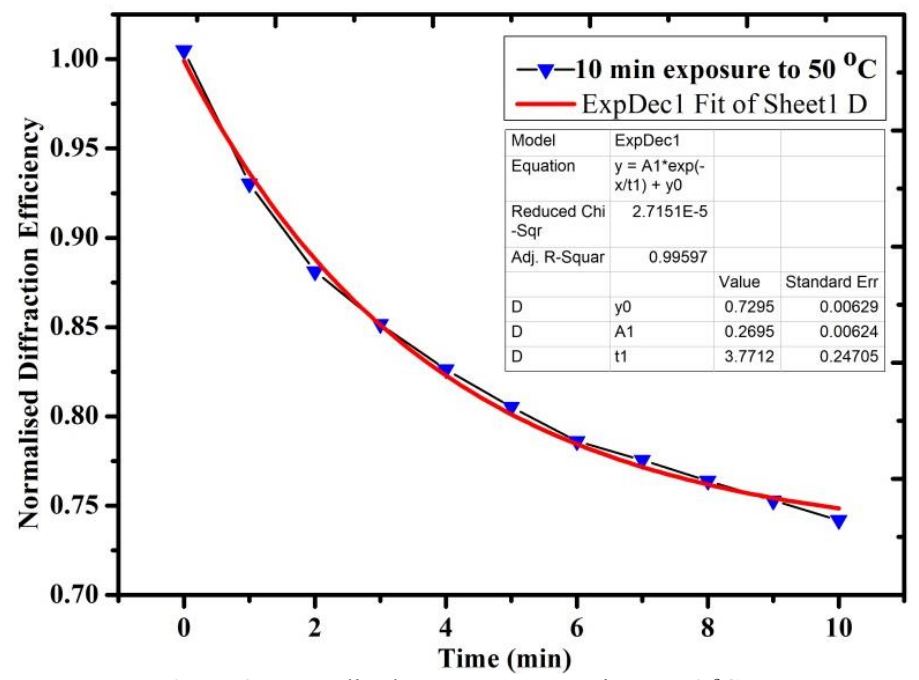

Figure 9. Normalised DE Vs exposure time at $50{ }^{\circ} \mathrm{C}$. 


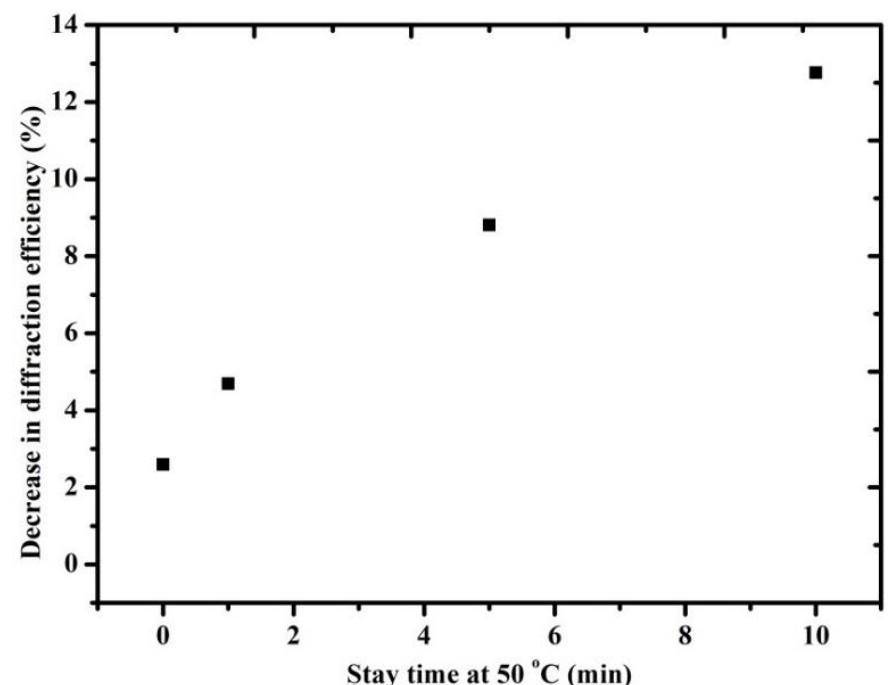

Figure 10. Irreversible decrease in DE (measured at $22{ }^{\circ} \mathrm{C}$ before and after exposure to $50{ }^{\circ} \mathrm{C}$ ) Vs time spent at $50{ }^{\circ} \mathrm{C}$

\section{CONCLUSION}

The temperature sensitivity of the un-slanted transmission phase holographic gratings recorded in NIPA-based photopolymer was studied. The DE of the holographic gratings are highly temperature dependent. The variation in $\mathrm{DE}$ depends upon the rate of temperature variation and the time spent at temperature above the LCST. The temperature response of the holographic transmission gratings can be used for the development of holographic temperature sensors operating in the temperature range from $22{ }^{\circ} \mathrm{C}$ to $50{ }^{\circ} \mathrm{C}$.

\section{ACKNOWLEDGMENT}

The authors would like to acknowledge the support by FOCAS Research Institute for providing research facilities and Dublin Institute of Technology Fiosraigh Postgraduate Scholarship Fund.

\section{REFERENCES}

[1] J. Ashley et al., "Holographic data storage," Ibm J. Res. Dev., vol. 44, no. 3, pp. 341-368, 2000.

[2] L. Dhar et al., "Holographic storage of multiple high-capacity digital data pages in thick photopolymer systems.," Opt. Lett., vol. 23, no. 21, pp. 1710-2, 1998.

[3] S. H. Lin, K. Y. Hsu, W.-Z. Chen, and W. T. Whang, "Phenanthrenequinone-doped poly(methyl methacrylate) photopolymer bulk for volume holographic data storage," Opt. Lett., vol. 25, no. 7, p. $451,2000$.

[4] L. Hesselink, S. S. Orlov, and M. C. Bashaw, "Holographic data storage systems," Proc. IEEE, vol. 92, no. 8, pp. 1231-1280, 2004.

[5] E. Fernández, M. Ortuño, S. Gallego, C. García, A. Beléndez, and I. Pascual, "Comparison of peristrophic multiplexing and a combination of angular and peristrophic holographic multiplexing in a thick PVA/acrylamide photopolymer for data storage," Appl. Opt., vol. 46, no. 22 , p. $5368,2007$.

[6] P. Macko and M. P. Whelan, "Fabrication of holographic diffractive optical elements for enhancing light collection from fluorescence-based biochips.," Opt. Lett., vol. 33, no. 22, pp. 2614-2616, 2008.

[7] A. K. Yetisen, I. Naydenova, F. Da Cruz Vasconcellos, J. Blyth, and C. R. Lowe, "Holographic sensors: Three-dimensional analyte-sensitive nanostructures and their applications," Chem. Rev., vol. 114, no. 20, pp. 10654-10696, 2014.

[8] M. Zawadzka et al., Photonic Materials for Sensing, Biosensing and Display Devices, vol. 229. 2016.

[9] D. Mao, Y. Geng, H. Liu, K. Zhou, L. Xian, and D. Yu, "Two-way shift of wavelength in holographic sensing of organic vapor in nanozeolites dispersed acrylamide photopolymer," Appl. Opt., vol. 55, no. 23, pp. 6212-6221, 2016.

[10] T. Mikulchyk, S. Martin, and I. Naydenova, "N-isopropylacrylamidebased photopolymer for holographic recording of thermosensitive transmission and reflection gratings," Appl. Opt., vol. 56, no. 22, p. 6348, 2017.

[11] I. Naydenova, R. Jallapuram, R. Howard, S. Martin, and V. Toal, "Investigation of the diffusion processes in a self-processing acrylamide-based photopolymer system.," Appl. Opt., vol. 43, no. 14, pp. 2900-2905, 2004.

[12] C. Carre and D. Lougnot, "Photopolymers for holographic recording from standard to self-processing materials", Journal de Physique III 3 (7), pp.1445-1460, JUL 1993.

[13] W. R. Klein and B. D. Cook, "Unified Approach to Ultrasonic Light Diffraction," IEEE Trans. on Sonics and Ultrasonics, pp. 123 - 134, 1967.

[14] H. Kogelnik, "Coupled Wave Theory for Thick Hologram Gratings," Bell Syst. Tech. J., vol. 48, no. 9, pp. 2909-2947, 1969. 